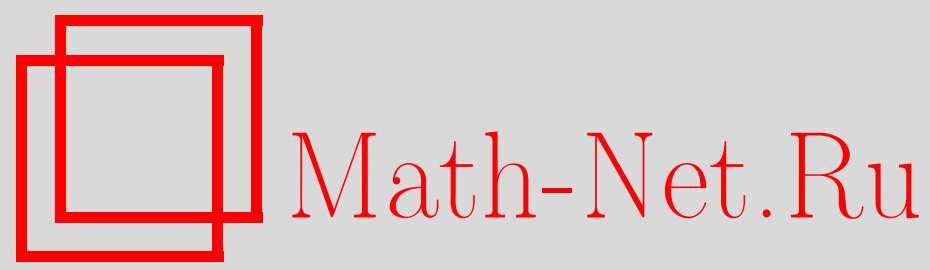

С. В. Пчелинцев, Специальность метабелевых алгебр Мальцева, Матем. заметки, 2003, том 74, выпуск 2, 257-266

DOI: https://doi.org/10.4213/mzm262

Использование Общероссийского математического портала Math-Net.Ru подразумевает, что вы прочитали и согласны с пользовательским соглашением http://www. mathnet.ru/rus/agreement

Параметры загрузки:

IP: 54.166 .219 .16

26 апреля 2023 г., 07:43:12

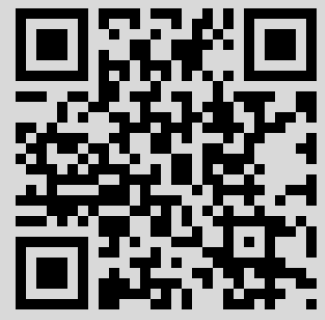




\title{
СПЕЦИАЛЬНОСТЬ МЕТАБЕЛЕВЫХ АЛГЕБР МАЛЬЦЕВА
}

\author{
С. В. Пчелинцев
}

Доказано, что для всякой метабелевой алгебры Мальцева $M$ над полем характеристики $\neq 2,3$ существует альтернативная алгебра $A$ такая, что алгебра $M$ вложима в коммутаторную алгебру $A^{(-)}$. При этом обертывающая алтернативная алгебра $A$ может быть найдена в многообразии алгебр с тождеством $[x, y][z, t]=0$. Доказательство указанного результата основано на построении аддитивньг базисов свободной метабелевой алгебры Мальцева и свободной альтернативной алгебры с тождеством $[x, y][z, t]=0$.

Библиография: 7 названий.

\section{Введение}

Алгебра Мальцева $M$ называется специальной, если она вложима в присоединенную алгебру $A^{(-)}$для подходящей альтернативной алгебры $A$. Вопрос о специальности произвольной алгебры Мальцева принадлежит Мальцеву. К настоящему времени доказана специальность полупервичных алгебр Мальцева [1] и алгебр Мальцева, удовлетворяющих тождествам простой нелиевой 7-мерной алгебры $C_{7}$ [2]. Отметим также, что ряд результатов о специальности некоторых идеалов мальцевских алгебр содержится в работе [3].

Данная заметка посвящена доказательству специальности любой метабелевой (разрешимой индекса 2) алгебры Мальцева над полем характеристики $\neq 2,3$. Заметка состоит из трех разделов. В разделе 1 доказьваются некоторые следствия тождества метабелевости. В разделе 2 строится аддитивный базис свободной метабелевой алгебры Мальцева. В разделе 3 доказана теорема о специальности - сначала для свободной метабелевой алгебры Мальцева, а затем для произвольной метабелевой алгебры Мальцева. Доказано, что для метабелевой алгебры Мальцева существует альтернативная обертывающая алгебра с тривиальным коммутантом ${ }^{1}$.

\section{1. Некоторые следствия метабелевости}

1.1. Определения, обозначения, тождества. Напомним основные определения.

Работа выполнена при финансовой поддержке Российского фонда фундаментальных исследований, грант № 0001-00339.

${ }^{1}$ Идеал с нулевым умножением называется тривиальным. 
ОпреДЕлЕниЕ. Антикоммутативная алгебра над полем $k$ характеристики, отличной от 2 и 3 , называется алгеброй Мальиева, если она удовлетворяет тождеству

$$
J(x, y, y z)=J(x, y, z) y
$$

где $J(x, y, z):=(x y) z+(y z) x+(z x) y$-якобиан элементов $x, y, z$.

Известно [4], что в алгебре Мальцева вьполнены тождества

$$
\begin{gathered}
x y^{2} z=x z y^{2}+x(y z) y+x y(y z), \\
x y z t+y z t x+z t x y+t x y z=(x z)(y t), \\
J(x y, z, t)+J(x z, y, t)+J(x, z, t) y+J(x, y, t) z=0, \\
2 J(x, y, z) t=J(x y, z, t)+J(y z, x, t)+J(z x, y, t), \\
J(x y, z, t)=x J(y, z, t)+J(x, z, t) y-2 J(x, y, z t),
\end{gathered}
$$

где, как обычно, отсутствующая расстановка скобок предполагается правонормированной, например, $x y^{2} z=((x y) y) z$.

ОПредЕЛЕниЕ. Алгебра $A$ над полем $k$ назьвается альтернативной, если в ней aсcоциатор $(x, y, z):=(x y) z-x(y z)$ является кососимметрической функцией от $x, y, z$.

Определив на аддитивной структуре алгебры $A$ новое умножение $[x, y]:=x y-y x$ (коммутатор элементов $x, y$ ), получим присоединенную или коммутаторную алгебру $A^{(-)}$. Из тождеств Муфанг вытекает, что если алгебра $A$ альтернативна, то коммутаторная алгебра $A^{(-)}$является алгеброй Мальцева.

ОПРЕДЕЛЕниЕ. Алгебраназьвается метабелевой, если в ней вьполнено тождество

$$
(x y)(z t)=0
$$

Всюду в дальнейшем, если не оговорено противное, используются следующие обозначения:

- $M$ - свободная метабелева алгебра Мальцева с множеством $X$ свободных порождающих над полем $k$ характеристики, отличной от 2 и 3 ;

- $a^{\prime}$ - оператор $R(a)$ умножения на элемент $a$, действующий на квадрате $M^{2}$;

- $J(M)$ - подпространство, порожденное якобианами $J(a, b, c)$;

- $E(M)$ - подпространство, порожденное элементами вида $a b c^{2}$;

- $Z(M)=\{z: J(z, M, M)=(0)\}$ - лиев центр алгебры $M$.

Из тождеств (2)-(6) вытекает, что подпространства $J(M), E(M), Z(M)$ являются идеалами произвольной алгебры Мальцева $M$, а не только метабелевой.

\section{2. Вспомогательные тождества алгебры $M$.}

Лемма 1. В алгебре $M$ справедливы следующие соотношения:

(a) $J\left(x y^{2}, x, z\right)=0$, в частности, $J\left(x y^{2}, y, z\right)=0$;

(б) $J\left(M^{2} y^{2}, z, t\right)=(0)$, m.e. $E(M) \subseteq Z(M)$;

(в) $J(M) t^{\prime 2}=0$. 
ДокАЗАтЕльство. (а) Заметим, что если $w \in M^{2}$, то на основании тождеств (3) и (7) справедливы равенства $J(w, y, z)=w\left[y^{\prime}, z^{\prime}\right]$ и $\left[y^{\prime} z^{\prime}, t^{\prime}\right]=0$. Значит, $J(w x, y, z)=$ $J(w, y, z) x$ ввиду тождества (6), и указанные функции кососимметричны по переменным $x, y, z$. В частности, имеем $J(w x, y, z)=J(w, x, y) z, J\left(x y^{2}, x, z\right)=J(x y y, x, z)=$ $J(x y, y, x) z=0$.

Тождество (б) получается линеаризацией тождества (a); (в) доказано в $[5, \S 3$, леммa $1, \Gamma)]$.

СлеДСТВИЕ. Если $w \in M^{2}$, то функиия $J\left(w x_{1} \ldots x_{n}, y, z\right)$ кососимметрична по переменным $x_{1}, \ldots, x_{n}, y, z$.

В дальнейшем это следствие используется без дополнительных пояснений.

Лемма 2. В алгебре Мальиева $М$ справедливо тожсдетво

$$
J(a, b, c) D=J(a D, b, c)+J(a, b D, c)+J(a, b, c D)-6 J(y z a, b, c),
$$

где оператор $D:=D(y, z)$ определяется равенством $a D=J(a, y, z)$.

ДокАЗАТЕЛЬСтво. Тождество (6) в терминах оператора $D$ принимает вид

$$
(a b) D=a \cdot b D+a D \cdot b-2 J(a, b, y z) \text {. }
$$

Отсюда на основании тождества метабелевости (7) имеем

$$
\begin{aligned}
J(a b c, y, z) & =(a b c) D=(a b) \cdot c D+(a b) D \cdot c-2 J(a b, c, y z)=(a b) D \cdot c \\
& =(a \cdot b D+a D \cdot b-2 J(a, b, y z)) c=a(d D) c+(a D) b c-2 J(a, b, y z) c \\
& =a(b D) c+(a D) b c-2 J(y z a, b, c),
\end{aligned}
$$

т.e.

$$
J(a b c, y, z)=a(b D) c+(a D) b c-2 J(y z a, b, c) .
$$

Переставляя циклически переменные $a, b, c$ и складьвая полученные три равенства, получаем на основании (7) требуемое тождество.

1.3. Однородные компоненты якобиана $J(M)$ типа $[3,1, \ldots]$ и $[2,2, \ldots]$. Мы считаем известными основные понятия, связанные с тождествами и свободными алгебрами. Все необходимые факты можно найти в монографиях $[6$, гл. 2] и [7, гл. 1].

ЛЕмма 3. Однородный многочлен алгебры $M$ степени $[3,1, \ldots]$ или $[2,2, \ldots]$ содержится в идеале $E(M)$.

ДокАЗАТЕЛЬСтво. Рассматривая факторалгебру $M / E(M)$, можно считать, что в метабелевой алгебре выполнено тождество

$$
x y z^{2}=0
$$

а также его линеаризация

$$
x y\left(z^{\prime} \circ t^{\prime}\right)=0, \quad \text { где } a \circ b=a b+b a .
$$


Тогда, во-первых, $x y\left(x^{\prime} \circ y^{\prime}\right)=0$ в силу тождества $(9)$, а, во-вторых, в силу бинарной лиевости алгебры Мальцева $x y\left[x^{\prime}, y^{\prime}\right]=0$, значит,

$$
x y^{2} x=0 \text {. }
$$

Докажем, что одночлен $y$ типа $[2,2, \ldots]$ равен нулю (одночлены типа $[3,1, \ldots]$ рассматриваются аналогично). Пусть, от противного, ненулевой одночлен $v$ имеет степень 2 по каждой из переменных $x$ и $y$. Если полная степень одночлена $v$ равна 4 , то утверждение вытекает из тождества (10). Допустим, что одночлен $v$ имеет вид $a b y_{1} \cdots y_{n}$, где $n \geqslant 3$ и $a, b, y_{1}, \ldots, y_{n}$ - переменные, среди которых два раза встречается каждая из букв $x$ и $y$. Если среди $y_{1}, \ldots, y_{n}$ дважды встречается одна буква, то в силу тождеств (8) и (9) получаем $v=0$. Итак, можно считать, что среди переменных $y_{1}, \ldots, y_{n}$ каждая из букв $x$ и $y$ встречается ровно один раз. Без ограничения общности, ввиду тождества (9) можно считать, что $y_{1}=y, y_{2}=x$. Следовательно, одна из букв $a, b$ совпадает с $x$, а другая с $y$, и на основании тождества (10) имеем

$$
v=a b y_{1} \cdots y_{n}= \pm x y^{2} x y_{3} \cdots y_{n}=0 \text {. }
$$

Противоречие.

ЛЕмма 4. Всякий однородный многочлен из якобиана $J(M)$ степени $[3,1, \ldots]$ или $[2,2, \ldots]$ является нулевым.

ДокАЗАТЕЛЬСТво. Поскольку идеал $J(M)$ линейно порождается якобианами, то утверждение достаточно доказать для якобианов $J(p, q, r)$, в которых $p, q, r$ - одночлены над $X$. В силу метабелевости можно считать, что $q, r \in X$. Из бинарной лиевости алгебры Мальцева вытекает, что одночлен $p$ имеет степень $\geqslant 3$. Значит, на основании следствия из леммы 1 можно считать, что одночлен $p$ имеет тип $[3,1, \ldots]$ или $[2,2, \ldots]$. Применяя теперь леммы 3 и 1 , получаем, что $p \in E(M) \subseteq Z(M)$, т.е. $J(p, q, r)=0$.

\section{2. Аддитивный базис алгебры $M$}

Будем считать в дальнейшем, что множество $X=\left\{x_{1}, x_{2}, \ldots\right\}$ свободных порождающих алгебры $M$ упорядочено по возрастанию индексов. Укажем аддитивный базис алгебры $M$, являющийся объединением базиса $B(L)$ свободной метабелевой алгебры Ли $L$ и базиса $B(J(M))$ якобиана $J(M)$. Хорошо известно [6, гл. 4], что одночлены

$$
x y t_{1} \cdots t_{n}, \quad \text { где } x, y, t_{i} \in X, \quad x<y, \quad x \leqslant t_{1} \leqslant t_{2} \leqslant \cdots,
$$

составляют базис $B(L)$ алгебры Ли $L$.

2.1. Правильные $J$-слова. Поскольку однородные многочлены из якобиана $J(M)$ степени $[3,1, \ldots]$ и $[2,2, \ldots]$ равны нулю (в силу леммы 4$),$ а характеристика поля $\neq 2,3$, то для описания аддитивной структуры алгебры $M$ достаточно указать базис пространства полилинейных элементов, содержашихся в якобиане $J(M)$.

ОПРЕДЕЛЕНИЕ. Правильны.ми $J$-словами над множеством $X_{n}=\left\{x_{1}, x_{2}, \ldots, x_{n}\right\}$, $n \geqslant 3$, называются следуюшие многочлены:

при $n=3: J\left(x_{1}, x_{2}, x_{3}\right)$;

при $n=4: J\left(x_{2}, x_{3}, x_{4}, x_{1}\right), J\left(x_{1} x_{p}, x_{q}, x_{r}\right), q<r$;

при $n=5: J\left(x_{1}\left(x_{2} x_{3}\right), x_{4}, x_{5}\right), J\left(\left[x_{1}, x_{2}, x_{3}\right], x_{4}, x_{5}\right), J\left(J\left(x_{p}, x_{2}, x_{3}\right), x_{q}, x_{r}\right), q<r$; при $n \geqslant 6: J\left(x_{1}\left(x_{2} x_{3}\right) x_{4} \ldots x_{n-2}, x_{n-1}, x_{n}\right), J\left(\left[x_{1}, x_{2}, x_{3}\right] x_{4} \ldots x_{n-2}, x_{n-1}, x_{n}\right)$, $J\left(J\left(x_{p}, x_{2}, x_{3}\right) x_{q_{1}} \ldots x_{q_{n-5}}, x_{r}, x_{s}\right), q_{1}<\cdots<q_{n-5}<r, s$, где $[x, y, z]:=x y z+x z y$. 
ЛЕмма 5. Правильные J-слова линейно независимы в коммутаторной алгебре Дорофеева.

ДокАЗАтЕльство. Пусть $D$ - альтернативная алгебра Дорофеева [7, гл. 6], т.е. разрешимая индекса 2 , но не нильпотентная альтернативная алгебра. Легко проверить, что в алгебре $D$ справедливы тождества $[[a b, x], x]=0, J^{-}(x, y, z)=6(x, y, z)$, где $J^{-}(x, y, z)$ - якобиан в присоединенной алгебре $D^{(-)}$. Следовательно, $[[a b, x], y]=$ $3 a b x y$. Отсюда вытекает, что в алгебре $D^{(-1)}$ не вьполняются тождества

$$
J\left(x y^{2} x_{1} \cdots x_{n}, z, t\right)=0, \quad n \geqslant 0 .
$$

Допустим теперь, что в алгебре $D^{(-)}$правильные $J$-слова степени 5 удовлетворяют соотношению

$$
\begin{aligned}
& \alpha J\left(x_{1}\left(x_{2} x_{3}\right), x_{4}, x_{5}\right)+\beta J\left(\left[x_{1}, x_{2}, x_{3}\right], x_{4}, x_{5}\right)+\gamma_{1} J\left(J\left(x_{1}, x_{2}, x_{3}\right), x_{4}, x_{5}\right) \\
& \quad+\gamma_{4} J\left(J\left(x_{4}, x_{2}, x_{3}\right), x_{1}, x_{5}\right)+\gamma_{5} J\left(J\left(x_{5}, x_{2}, x_{3}\right), x_{1}, x_{4}\right)=0 .
\end{aligned}
$$

Считая $w \in[D, D]$ иполагая последовательно $x_{1}=w, x_{4}=w, x_{5}=w$, получаем $\gamma_{1}=0$, $\gamma_{4}=0, \gamma_{5}=0$. Значит, $\left.\left.\alpha J\left(x_{1}\left(x_{2} x_{3}\right), x_{4}, x_{5}\right)\right)+\beta J\left(\left[x_{1}, x_{2}, x_{3}\right], x_{4}, x_{5}\right)\right)=0$. Полагая $x_{2}=x_{3}$, получим в алгебре $D^{(-)}$тождество вида $2 \beta J\left(x y^{2}, z, t\right)=0$, или после линеаризации имеем $\beta J^{-}([[a b, x], y], z, t)=0$. Тогда $\beta a b x y z t=0$ и, поскольку алгебра $D$ не нильпотентна, то $\beta=0$, а значит, и $\alpha=0$. Тем самым, доказана линейная независимость правильных $J$-слов степени 5 . Линейная независимость остальных правильных $J$-слов доказьвается аналогично; заметим, что линейная независимость правильных $J$-слов степени $n \geqslant 6$ доказана в [5, 33 , лемма 4].

\section{2. Аддитивный базис якобиана $J(M)$.}

ЛЕмма 6. Всякий однородный многочлен степени $[1,1, \ldots, 1]$ над множсеством $X_{n}, n \geqslant 3$, из якобиана $J(M)$ представим в виде линейной комбиначии правильных $J$-слов.

ДокАЗАТЕЛЬСТво. Если $n=3$ или $n=4$, то требуемое утверждение легко вытекает из кососимметричности якобиана и полной линеаризации тождества $J(x y, x, y)=0$. Если $n \geqslant 6$, то утверждение вытекает из [5, § 3 , лемма 3$]$.

Итак, пусть $n=5 ; X_{5}=\{x, y, z, t, u\}, x<y<z<t<u$. Рассмотрим сначала полилинейньй многочлен $j$ над $X_{5}$ вида $j\left(t_{1}, t_{2}, t_{3}, t_{4}, t_{5}\right)=J\left(\left[t_{1}, t_{2}, t_{3}\right], t_{4}, t_{5}\right)$, где $t_{i}$ обозначают переменные из множества $X_{5}$. Пусть $V$ - линейное подпространство, порожденное правильными $J$-словами над $X_{5}$, т.е. многочленами вида $J(x(y z), *, *), J([x, y, z], *, *)$, $J(J(*, y, z), *, *)$, где через * обозначены переменные из множества $X_{5}$.

Заметим, что функция $J\left(\left[t_{1}, t_{2}, t_{3}\right], t_{4}, t_{5}\right)$ симметрична относительно $t_{2}$ и $t_{3}$; ввиду леммы 1 она кососимметрична по переменным $t_{1}, t_{4}, t_{5}$, и, кроме того, справедливы тождества

$$
\begin{gathered}
J([a, b, c], p, q)+J([p, b, c], a, q)=0, \\
J([a, b, c], p, q)+J([a, c, p], b, q)+J([a, p, b], c, q)=0 .
\end{gathered}
$$

Отсюда вытекает, что многочлен $j$ является линейной комбинацией слов $J([*, y, *], *, *)$. Каждый из элементов вида $J([*, y, *], *, *)$ ввиду аналогичных соображений является 
линейной комбинацией элементов $J([x, y, *], *, *)$ и $J([*, y, x], *, *)$. Тогда, без ограничения общности, достаточно рассмотреть следующие две возможности:

I) $j=J([z, y, x], *, *)$,

II) $j=J([x, y, *], z, *)$.

Рассмотрим последовательно каждьй из этих случаев.

I) Случай $j=J([z, y, x], *, *)$. Поскольку $2 x y z=x(y z)+[x, y, z]+J(x, y, z)$, то для полилинейного одночлена $w(x, y, z)$ верно соотношение

$$
J(w(x, y, z), *, *) \in V,
$$

в частности, $j \equiv 0$ (здесь и далее знак $\equiv$ означает сравнение по модулю $V$ ).

II) Случай $j=J([x, y, p], z, q)$.

Рассмотрение этого случая представим в виде последовательности шагов.

Шаг 1. Докажем, что $2 J\left(x b^{2}, y, z\right)=2 J(x b, y, z) b=-J(J(b, y, z), x, b)$.

Действительно, ввиду леммы 2 и тождества (6) имеем

$$
\begin{aligned}
2 J\left(x b^{2}, y, z\right) & =2 J(x b, y, z) b=2(x J(b, y, z)+J(x, y, z) b-2 J(x, b, y z)) b \\
& =-2 J(b, y, z) x^{\prime} b^{\prime}=-J(b, y, z)\left[x^{\prime}, b^{\prime}\right]-J(b, y, z)\left(x^{\prime} \circ b^{\prime}\right) \\
& =-J(J(b, y, z), x, b) .
\end{aligned}
$$

Шаг 2. Докажем, что $J([x, p, q], y, z) \equiv 0$.

Тождество из шага 1 может быть записано в виде $J([x, b, b], y, z)=-J(J(b, y, z), x, b)$. Проведя его линеаризацию, получаем

$$
2 J([x, p, q], y, z)=-J(J(p, y, z), x, q)-J(J(q, y, z), x, p) \equiv 0,
$$

поскольку $J(J(*, y, z), *, *) \equiv 0$. Тем самым, требуемое сравнение доказано.

Шаг 3. Функция $j=J([x, y, p], z, q)$ кососимметрична (по модулю $V$ ) относительно $p$ и $q$, а также кососимметрична относительно $y$ и $z$, т.е. справедливы сравнения

$$
\begin{aligned}
& J([x, y, p], z, q)+J([x, y, q], z, p) \equiv 0 \\
& J([x, y, p], z, q)+J([x, z, p], y, q) \equiv 0 .
\end{aligned}
$$

Действительно,

$$
\begin{aligned}
0 & =J([x, y, p], z, q)+J([x, p, q], z, y)+J([x, q, y], z, p) \text { ввиду }(12) \\
& \equiv J([x, y, p], z, q)+J([x, q, y], z, p) \quad \text { ввиду шага } 2 \\
& =J([x, y, p], z, q)+J([x, y, q], z, p) \\
0 & =J([x, y, p], z, q)+J([x, p, z], y, q)+J([x, z, y], q, p) \quad \text { ввиду }(12) \\
& \equiv J([x, y, p], z, q)+J([x, p, z], y, q) \quad \text { ввиду }(13) \\
& =J([x, y, p], z, q)+J([x, z, p], y, q)
\end{aligned}
$$

Шаг 4. Докажем, что $J(x p y, z, q) \equiv-J(x q y, z, p)$. Действительно,

$$
2 J(x b y, z, b)=2 J(x b, y, z) b=-J(J(b, y, z), x, b)
$$

в силу равенства из шага 1 . Проведя линеаризацию ввиду сравнения $J(J(*, y, z), *, *) \equiv 0$, получаем требуемое сравнение. 
Шаг 5. Докажем, что $J(J(x, p, q), y, z) \equiv 0$. Действительно, ввиду леммы 2 имеем

$$
\begin{aligned}
J(J(x, p, q), y, z)= & J(J(x, y, z), p, q)+J(x, J(p, y, z), q) \\
& +J(x, p, J(q, y, z))-6 J(y z x, p, q) \equiv 0,
\end{aligned}
$$

поскольку $J(J(*, y, z), *, *) \equiv 0$ и $J(x(y z), *, *) \equiv 0$.

Шаг 6. Докажем, что $J(x(p q), y, z) \equiv 0$. Заметим сначала, что

$$
J(x(a b), a, b)=-J(a(a b), x, b)=J\left(b a^{2}, b, x\right)=0
$$

в силу леммы 1 . Используя линеаризацию тождества $J(a(a b), a, b)=0$, имеем в силу соотношения (13) и шага 4

$$
\begin{aligned}
-J(x(p q), y, z) & =J(x(y q), p, z)+J(x(p z), y, q)+J(x(y z), p, q) \\
& \equiv J(x(y q), p, z)+J(x(p z), y, q) \equiv-J(x(y p), q, z)+J(x(p z), y, q) \\
& =J(z(y p), q, x)-J(y(p z), x, q)=J(p y z-p z y, q, x) \\
& =J(J(p, y, z)+p(y z), q, x)=J(J(p, y, z), q, x)+J(x(y z), p, q) \\
& \equiv J(J(p, y, z), q, x) \equiv 0 .
\end{aligned}
$$

Теперь мы можем завершить проверку сравнения $j \equiv 0$, где $j=J([x, y, p], z, q)-$ якобиан из случая II. Ввиду соотношения (13) и сравнений из шагов $2,5,6$ имеем

$$
\begin{aligned}
2 j & =2 J([x, y, p], z, q)=2 J(x y p+x p y, z, q)=-2 J(x y z, p, q)+2 J(x p y, z, q) \\
& \equiv 2 J(x p y, z, q)=2 J(x p q, y, z)=J([x, p, q]+J(x, p, q)+x(p q), y, z) \equiv 0 .
\end{aligned}
$$

Итак, утверждение леммы доказано для якобианов вида $J([*, *, *], *, *)$.

Тогда по модулю $V$ функция $J\left(t_{1} t_{2} t_{3}, t_{4}, t_{5}\right)$, где $t_{i}$ - переменные из $X_{5}$, кососимметрична по всем переменным, и, значит, содержится в пространстве $V$.

ЗАмЕчАнИЕ. Повторив доказательство леммы 6 , легко понять, что всякий многочлен типа $[1,2,1, \ldots, 1]$ из $J(M)$ пропорционален элементу $J\left(x_{1} x_{2}^{2} x_{3} \ldots x_{n-2}, x_{n-1}, x_{n}\right)$, $n \geqslant 5$. Следовательно, указанные элементы вместе с правильньми $J$-словами образуют базис $J(M)$.

\section{3. Вложение метабелевых алгебр Мальцева в альтернативные алгебры}

3.1. Специальность свободной метабелевой алгебры Мальцева. Пусть поле $K$ является расширением поля $k$. Тогда алгебра $K \otimes_{k} M$ является свободной метабелевой алгеброй Мальцева над полем $K$. Поэтому в дальнейшем можно считать, что поле $k$ бесконечно, а любое многообразие $k$-алгебр однородно.

Свободная метабелева $k$-алгебра $M$ обладает базисом $B(L) \cup B(J(M))$ (см. раздел 2). Далее, легко понять, что элементы из $B(L)$ линейно независимы в 2-мерной неабелевой алгебре Ли, которая изоморфна алгебре $C^{(-)}$, где $C$ - ассоциативная алгебра матриц

$$
\left(\begin{array}{ll}
a & b \\
0 & 0
\end{array}\right), \quad \text { где } a, b \in k .
$$

В разделе 2 было доказано, что многочлены из $B(J(M))$ линейно независимы в коммутаторной алгебре Дорофеева $D^{(-)}$, следовательно, элементы из $B(L) \cup B(J(M))$ линейно независимы в коммутаторной алгебре $(C \oplus D)^{(-)}$. Обозначим через $F$ свободную алгебру над $X$ в многообразии, порожденном алгебрами $C$ и $D$. Тогда из предыдущего получаем, что свободная метабелева алгебра Мальцева $M$ (над $X$ ) изоморфна подалгебре алгебры $F^{(-)}$, порожденной множеством $X$. Следовательно, свободная метабелева алгебра Мальцева специальна. 
3.2. Одно замечание об альтернативной алгебре, в которой выполнено тож,деством $[x, y][z, t]=0$. Поскольку алгебры $C$ и $D$ удовлетворяют тождеству

$$
[x, y][z, t]=0
$$

то в качестве альтернативной обертьвающей алгебры для $M$ можно взять свободную альтернативную алгебру $A$ с единицей, удовлетворяющую тождеству (14).

ЛЕмма 7. Пусть $A$ - альтернативная алгебра, удовлетворяющая тождеству (14). Тогда ее коммутант $A^{\prime}$ является тривиальным идеалом.

ДокАЗАтЕльство. Поскольку в алгебре $A$ верно тождество $J^{-}(x, y, z)=6(x, y, z)$, то коммутант представим в виде $A^{\prime}=[A, A] A$. Далее, если $v, w$ - коммутаторы, то $(v, w, a)=(1 / 6) J^{-}(v, w, a)=0$, откуда имеем

$$
w(v a)=(w v) a=0, \quad(v a) w=(a v+[v, a]) w=(a v) w=a(v w)+(a, v, w)=0,
$$

т.е. $[A, A] \subseteq \operatorname{Ann} A^{\prime}$, значит, $A^{\prime 2}=(0)$.

3.3. Аддитивный базис альтернативной алгебры, в которой выполнено тождество $[x, y][z, t]=0$. Всюду ниже $A$ - свободная альтернативная алгебра с тождеством (14), $X$ - множество свободных порождаюших, $M$ - подалгебра алгебры $A^{(-)}$, порожденная множеством $X$.

ЛЕмма 8. Если $y_{1}, y_{2}, \ldots$ - базис пространства $[M, M]$, то алгебра $A$ обладает аддитивным базисом над полем $k$, состоящим из әлементов вида
a) $x_{i_{1}} \circ x_{i_{2}} \circ \cdots \circ x_{i_{n}}$
б) $y_{p} \circ\left(x_{i_{1}} \circ x_{i_{2}} \circ \cdots \circ x_{i_{n}}\right)$,

әде $i_{1} \leqslant i_{2} \leqslant \cdots \leqslant i_{n}, n \geqslant 0^{2} ;$ отсутствующая расстановка скобок правонормирована.

ДокАЗАТЕльСтво. Докажем сначала индукцией по степени одночлена, что всякий одночлен является линейной комбинацией многочленов типа а) и

$$
\text { в) } y \circ\left(x_{i_{1}} \circ x_{i_{2}} \circ \cdots \circ x_{i_{n}}\right) \text {, }
$$

где $y$ - однородный многочлен из $[M, M], i_{1} \leqslant i_{2} \leqslant \cdots \leqslant i_{n}$.

Пусть $A^{\prime}$ - коммутант алгебры $A$. Факторалгебра $A / A^{\prime}$ является свободной ассоциативно-коммутативной алгеброй, т.е. $A / A^{\prime}$ - алгебра многочленов, в которой элементы

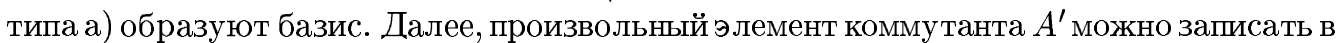
виде линейной комбинации элементов $v$ вида $v=[p, q] r$, где $p, q, r$-одночлены. Каждьй из одночленов $p, q$ по предположению индукции представим в виде линейной комбинации элементов типа а) и в). Поскольку коммутатор является йордановым дифференцированием, то элемент $v$ является линейной комбинацией элементов (того же состава, что и $v$ ) вида $w T\left(v_{1}\right) \ldots T\left(v_{s}\right)$, где $w$ - однородный элемент из $[M, M], v_{1}, \ldots, v_{s}$ - одночлены над $X, T(v)$ - оператор правого или левого умножения в алгебре $A$ на элемент $v$.

Докажем, что элемент $\xi$ вида $w T\left(v_{1}\right) \cdots T\left(v_{s}\right)$ при указанных ограничениях на элементы $w, v_{1}, \ldots, v_{s}$, представим в виде линейной комбинации элементов типа в). Для

${ }^{2}$ Для $n=0$ слово типа а) считается равным единице 1 , а слово типа б) - элементу $y_{p}$. 
этого рассмотрим вспомогательньй элемент $t$ вида $t=x T\left(v_{1}\right) \cdots T\left(v_{s}\right)$, считая, что переменная $x$ (из множества $X$ ) больше всех переменных, входящих в состав многочлена $\xi$. По индукции элемент $t$ представим в виде линейной комбинации базисных элементов, относительно переменных, входящих в слово $t$. Подставим в полученную линейную комбинацию вместо переменной $x$ элемент $w$. При этой подстановке элемент $t$ перейдет в $\xi$; базисные слова типа а) перейдут в слова типа в), а базисные слова типа в) либо перейдут в нулевые, либо в элементы вида в).

Тем самьм, доказано, что элементы вида а) и в), линейно порождают пространство $A$. Значит, тем же свойством обладают элементы вида а) и б).

Теперь докажем линейную независимость элементов типа а) и б). Для этого достаточно ограничиться рассмотрением элементов типа б). Пусть для некоторых ненулевых скаляров верно равенство $\sum_{p} \alpha_{p} y_{p} \circ f_{p}=0$, где $f_{p}$ - одночлены типа а). Среди всех одночленов типа $f_{p}$ выберем одночлены, имеющие максимальную полную степень, а среди них выберем наибольший (в смысле лексикографики) одночлен

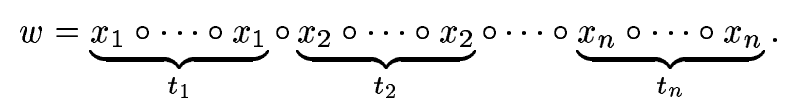

Обозначая, как обычно, через $\Delta(x)$ оператор частной производной по переменной $x[7]$ и применяя к тождеству $\sum_{p} \alpha_{p} y_{p} \circ f_{p}=0$ оператор $\Delta^{t_{1}}\left(x_{1}\right) \Delta^{t_{2}}\left(x_{2}\right) \ldots \Delta^{t_{n}}\left(x_{n}\right)$, получаем $\sum_{p} 2^{N} \alpha_{p} y_{p}=0$ (степень двойки появляется за счет йордановых произведений), суммирование ведется по тем индексам $p$, для которых верно равенство $f_{p}=w$. Поскольку элементы $y_{p}$ линейно независимы, то хотя бы одно из чисел $\alpha_{p}$ равно нулю, что невозможно по предположению.

СлЕдСТВИЕ. а) Элементы типа б) из леммы 8 образуют аддитивный базис коммутанта $A^{\prime}$.

б) Имеет место равенство $M \cap A^{\prime}=[M, M]$.

3.4. Основной результат. Нам потребуется следующее уточнение леммы 8 .

ЛЕмма 9. Пусть I - идеал алгебры $M ; h_{1}, h_{2}, \ldots$ - аддитивный базис пространства $I \cap[M, M]$. Дополним эту систему до базиса пространства I әлементами $g_{1}, g_{2}, \ldots$. Пусть также $f_{1}, f_{2}, \ldots$ - дополнение системы $h_{1}, h_{2}, \ldots$ до базиса пространства $[M, M] ; e_{1}, e_{2}, \ldots$ - дополнение системы $f_{1}, f_{2}, \ldots, g_{1}, g_{2}, \ldots, h_{1}, h_{2}, \ldots$ до базиса пространства $M$. Тогда алгебра $A$ обладает аддитивным базисом над полем $k$, состояшим из әлементов вида:

a) $a_{i_{1}} \circ a_{i_{2}} \circ \cdots \circ a_{i_{n}}$,

б) $y \circ\left(a_{i_{1}} \circ a_{i_{2}} \circ \cdots \circ a_{i_{n}}\right)$,

әде все $a_{i}$ принадлежст упорядоченному множеству $e_{1}<e_{2}<\cdots<g_{1}<g_{2}<\cdots$; $i_{1} \leqslant i_{2} \leqslant \cdots, n \geqslant 0 ; y \in\left\{f_{1}, f_{2}, \ldots, h_{1}, h_{2}, \ldots\right\}$.

ДоКАЗАТЕЛЬСтво. В силу следствия из леммы 8 вытекает, что система многочленов типа а) и б) линейно порождает обертьвающую алгебру $A$. Для доказательства линейной независимости указанных элементов рассмотрим канонические гомоморфизмы $-: A \rightarrow A / A^{\prime},{ }^{*}: M \rightarrow M /[M, M]$. В силу следствия из леммы 8 гомоморфизм * является ограничением на $M$ гомоморфизма $\cdot$.

Поскольку элементы $\bar{e}_{1}, \bar{e}_{2}, \ldots, \bar{g}_{1}, \bar{g}_{2}, \ldots$ образуют базис пространства $k \bar{X}$, то факторалгебра $A / A^{\prime}$ изоморфна алгебре многочленов $k\left[\bar{e}_{1}, \bar{e}_{2}, \ldots, \bar{g}_{1}, \bar{g}_{2}, \ldots\right]$. Учитьвая, 
что образы слов типа а) при гомоморфизме · линейно независимы, получаем линейную независимость самих слов типа а). Тогда, без ограничения общности, можно считать, что линейно зависимы слова типа б). Каждьй из элементов $y \circ\left(a_{i_{1}} \circ a_{i_{2}} \circ \cdots \circ a_{i_{n}}\right)$ на основании леммы 7 имеет вид $y \circ\left(a_{i_{1}}^{*} \circ a_{i_{2}}^{*} \circ \cdots \circ a_{i_{n}}^{*}\right)$, где образ $a^{*}$ получен из $a$ отбрасьванием однородных компонент степени большей 1. Значит, мы получаем линейную зависимость элементов $y \circ\left(v_{i_{1}} \circ v_{i_{2}} \circ \cdots \circ v_{i_{n}}\right)$, где $\left(v_{s}\right)$ - базис пространства $k X$. Пусть $\varphi$ - автоморфизм алгебры $A$, продолжающий отображение $x_{s} \varphi=v_{s}$. Тогда под действием $\varphi^{-1}$ многочлены вида $y \circ\left(v_{i_{1}} \circ v_{i_{2}} \circ \cdots \circ v_{i_{n}}\right)$ перейдут в базисные элементы типа б) из леммы 8. Тем самьм, линейно зависимыми оказались базисные слова из леммы 8, что, очевидно, невозможно.

ТЕоремА. Всякая метабелева алгебра Мальцева над полем характеристики, отличной от 2 и 3 , специальна.

ДокАЗАТЕЛьСТво. Покажем вначале, что для произвольного идеала $I$ алгебры $M$ верно равенство $I^{\wedge} \cap M=I$, где $I^{\wedge}$ - идеал, порожденньй множеством $I$ в $A$.

В силу леммы 9 легко понять, что идеал $I^{\wedge}$ линейно порождается элементами $a_{i_{1}} \circ a_{i_{2}} \circ \cdots \circ a_{i_{n}}, y \circ\left(a_{i_{1}} \circ a_{i_{2}} \circ \cdots \circ a_{i_{n}}\right)$, в запись каждого из которых входит хотя бы один базисньй элемент идеала $I$. Если некоторая линейная комбинация указанных элементов содержится в алгебре Мальцева $M$, то получаем равенство

$$
\begin{aligned}
& \sum \beta a_{i_{1}} \circ a_{i_{2}} \circ \cdots \circ a_{i_{n}}+\sum \gamma y \circ\left(a_{i_{1}} \circ a_{i_{2}} \circ \cdots \circ a_{i_{n}}\right) \\
& =\sum \lambda e+\sum \mu f+\sum \nu g+\sum \eta h .
\end{aligned}
$$

В силу леммы 9 в правой части этого равенства векторы типа $e$ и $f$ могут встречаться только с нулевыми коэффищиентами. Значит, в правой части последнего равенства находятся только элементы из идеала $I$.

Доказанное равенство $I^{\wedge} \cap M=I$ позволяет завершить доказательство теоремы. Образ алгебры $M$ при каноническом гомоморфизме $A \rightarrow A / I^{\wedge}$ совпадает с алгеброй $\left(M+I^{\wedge}\right) / I^{\wedge}$ изоморфной алгебре $M /\left(I^{\wedge} \cap M\right)=M / I$. Следовательно, алгебра $M / I$ вложима в присоединенную алгебру $\left(A / I^{\wedge}\right)^{(-)}$, т.е. является специальной.

\section{СПИСОК ЦИТИРОВАННОЙ ЛИТЕРАТУРЫ}

[1] Филиппов В.Т.Первичные алгебры Малцева // Матем. заметки. 1982. Т. 31. №5. C. $669-678$.

[2] Sverchkov S. Varieties of special algebras // Comm. Algebra. 1988. V. 16. P. 1877-1919.

[3] Филиппов В. Т. О вложении мальцевских алгебр в альтернативные // Алгебра и логика. 1983. T. 22. № 4. C. $443-465$.

[4] Sagle A. A. Malcev algebras // Trans. Amer. Math. Soc. 1961. V. 101. № 3. P. 426-458.

[5] Пчелинцев С. В. Разрешимые индекса 2 многообразия алгебр // Матем. сб. 1981. Т. 115. № 2. C. 179-203.

[6] Бахтурин Ю. А. Тождества в алгебрах Ли. М.: Наука, 1985.

[7] Жевлаков К. А., Слинько А. М., Шестаков И. П., Ширшов А. И. Кольца, близкие к ассоциативным. М.: Наука, 1978. 